\title{
DIAGNÓSTICO DA UROLITÍASE EM FELINOS
}

\author{
Veridiane da Rosa Gomes ${ }^{1}$, Paula Costa Ariza ${ }^{2}$, Layla Lívia de Queiroz ${ }^{2}$, Victor \\ Gerardo Petro Hernandez ${ }^{3}$, Maria Clorinda Soares Fioravanti ${ }^{4}$ \\ ${ }^{1}$ Doutoranda em Ciência Animal, Escola de Medicina Veterinária e Zootecnia, \\ Universidade Federal de Goiás, Goiânia, Brasil, email: \\ veridiane.rgomes@yahoo.com.br \\ ${ }^{2}$ Doutoranda em Ciência Animal, Escola de Medicina Veterinária e Zootecnia, \\ Universidade Federal de Goiás, Goiânia, Brasil \\ ${ }^{3}$ Mestre em Ciência Animal, Escola de Medicina Veterinária e Zootecnia, \\ Universidade Federal de Goiás, Goiânia, Brasil \\ ${ }^{4}$ Doutora, docente da Escola de Medicina Veterinária e Zootecnia, Universidade \\ Federal de Goiás, Goiânia, Brasil
}

\section{Recebido em: 06/04/2019 - Aprovado em: 10/06/2019 - Publicado em: 30/06/2019 DOI: 10.18677/EnciBio_2019A54}

\begin{abstract}
RESUMO
O sistema urinário é acometido por diversas enfermidades que ocasionam sinais clínicos bastante similares. Dessa forma, a realização de exames complementares é importante para determinação da doença de base e instituição de medidas terapêuticas adequadas. Dentre as enfermidades urinárias observadas em felinos estão a cistite idiopática, urolitíase, tampões uretrais, anormalidades anatômicas, problemas comportamentais, neoplasias e infecções do trato urinário. $O$ uso de técnicas laboratoriais e de diagnóstico por imagem associadas ao histórico clínico, auxiliam no diagnóstico definitivo. Em casos de litíase urinária, tais exames além de indicarem a presença do urólito, ajudam a predizer o tipo mineral presente, pois, é com base na composição do cálculo que o tratamento e prevenção são instituídos. Entretanto, cabe ressaltar que a associação dessas análises para predizer é passível de erro, sendo indicadas somente quando não for possível a obtenção do cálculo. A presença de urólitos não deve ser encarada como diagnóstico final, mas o início de uma série de investigações visando entender a sua causa, que vão desde fatores alimentares até fatores genéticos. Portanto, a avaliação da composição do cálculo por meio de técnicas qualitativas e quantitativas são as formas mais seguras de proceder com as medidas necessárias. Diversos são os métodos disponíveis para determinar as substâncias presentes em uma amostra, cada uma apresenta vantagens e desvantagens. Objetivou-se com a presente revisão apresentar os métodos de diagnóstico da urolitíase em felinos, bem como descrever as técnicas recomendadas para determinar a composição dos cálculos.
\end{abstract}

PALAVRAS-CHAVE: espectroscopia, gato, técnicas de análise 


\title{
DIAGNOSIS OF FELINE UROLITHIASIS
}

\begin{abstract}
The urinary tract is afflicted by several diseases that run with similar clinical signs. Therefore, complementary exams are important for determining the underlying cause and deciding on the appropriate treatment. Among the urinary diseases in cats, idiopathic cystitis, urolithiasis, urethral buffers, anatomical abnormalities, behavioral issues, neoplasms and urinary tract infections (UTI) can be cited. Laboratory and diagnostic imaging techniques associated with clinical history, helps with the definitive diagnosis. In cases of urinary lithiasis, such tests, both indicates the presence of uroliths and help predicting the mineral type. Treatment and prevention are based on the composition of the calculus. However, it should be noted that the association of these analyses for composition prediction is subject to error, which may impair the therapy. That way, the prediction is indicated only when it is not possible to obtain and analyze the calculi. The identification of uroliths should not be considered as a final diagnosis, but the beginning of a series of investigations aiming to understand the cause of urinary lithogenesis, ranging from dietary to genetic factors. Therefore, the composition analysis of the calculi using qualitative and quantitative methods is the safest way to proceed. Several methods are available to determine the substances present in a sample, each one presenting advantages and disadvantages. The objective of this review is to present the different diagnosis methods of urolithiasis in felines and the characteristics observed in the tests that may suggest the type of substance present in the stone; as well as to describe the techniques recommended to determine the composition of the calculi.
\end{abstract}

KEYWORDS: cat, spectroscopy, urinary concretions

\section{INTRODUÇÃO}

O diagnóstico da urolitíase se baseia na anamnese, sinais clínicos, exame físico, exames laboratoriais e de imagem (OSBORNE et al., 1996; PALM; WESTROPP, 2011; PINEDA et al., 2015) Os urólitos são facilmente detectados em radiografias simples e/ou ultrassonografia abdominal (US), mas a tomografia computadorizada (TC) também é considerada um método diagnóstico, principalmente em pacientes humanos. As técnicas de imagem também auxiliam no diagnóstico diferencial (LANGSTON et al., 2008; TÜRK et al., 2016; NYKAMP, 2017).

Conforme a quantidade, tamanho, localização e sinais clínicos apresentados define-se a forma de obtenção dos cálculos, uma vez que para estabelecer a terapêutica correta deve-se conhecer a composição do urólito. Embora os exames complementares para o diagnóstico possam auxiliar a predizer o tipo mineral presente, são as análises qualitativas e quantitativas as indicadas para confirmar a composição (LULICH et al., 2011).

A classificação do cálculo baseia-se no mineral presente, os tipos observados são oxalato de cálcio (monohidratado e diidratado), estruvita (fosfato amônio magnesiano), urato (urato amônio, sais de urato, ácido úrico), xantina, cistina, fosfato de cálcio, sílica e sangue seco solidificado (OSBORNE et al., 1995; CANNON et al., 2007; HOUSTON et al., 2016).

Quanto à estrutura o urólito possui um nidus (núcleo), que é a região inicial de desenvolvimento (LULICH et al., 2011). Circundando o núcleo, tem-se a pedra, que é a maior porção da amostra, sendo que a camada mais externa é denominada parede (OSBORNE et 
al., 1995). Podem estar presentes os chamados cristais de superfície, circundando a parede de maneira incompleta. Essa divisão permite a classificação do cálculo quanto à quantidade de cada substância em cada camada. Por exemplo, quando $70 \%$ da amostra é composta por um tipo mineral, ele é classificado como simples. Em casos onde não é possível identificar camadas e nem um mineral atinge $70 \%$ do total, é chamado de misto. Quando possui camadas sobrepostas de diferentes tipos minerais é chamado composto (OSBORNE et al., 1995; LULICH et al., 2011; HOUSTON et al., 2016). O conhecimento da estrutura e dos tipos de litíase urinária é importante na análise das pedras.

\section{MÉTODOS DE DIAGNÓSTICO DA UROLITÍASE ANAMNESE E SINAIS CLÍNICOS}

A anamnese é uma parte importante no diagnóstico, pois os dados obtidos servem para sugerir a presença da litíase urinária (BARTGES; CALLENS, 2015). Diversas são as enfermidades que predispõem à urolitíase. O distúrbio deve sempre ser considerado em felinos com doença renal crônica (DRC), disfunção hepática, doenças que cursem com hipercalcemia e infecções urinárias recorrentes e em pacientes obesos, com histórico anterior ou histórico familiar da doença. (OSBORNE et al., 1995; OSBORNE et al., 1996; LULICH et al., 2016; GOMES et al., 2018).

A raça do animal acometido também é fator importante, visto que as raças Persa e Himalaia são predispostas ao desenvolvimento de urólitos, principalmente os compostos de estruvita e oxalato de cálcio, enquanto a raça Siamês apresenta predisposição aos cálculos de urato (CANNON et al., 2007; HOUSTON; MOORE, 2009). Com relação à faixa etária, felinos com menos de quatro anos são mais predispostos à urolitíase por estruvita. Dos quatro a sete anos a prevalência de estruvita e oxalato de cálcio é a mesma, entretanto a partir dos sete anos de idade a ocorrência de oxalato de cálcio é maior (CANNON et al., 2007).

Com relação ao sexo, de modo geral os machos são 1,6 vezes mais acometidos por cálculos de oxalato de cálcio, quando comparado às fêmeas. Contudo, fêmeas apresentam 1,2 vezes maior predisposição à estruvita (CANNON et al., 2007).

Os sinais clínicos de urolitíase em felinos variam de acordo com o número de urólitos presentes, localização no trato urinário e características físicas do urólito. A presença de um único urólito ou vários com superfície lisa na bexiga ou pelve renal podem ser menos irritantes à mucosa vesical que cálculos irregulares ou com bordas afiadas (PALM; WESTROPP, 2011; GRAUER, 2015).

Os urocistólitos (cálculos vesicais) ocasionam irritação da parede da bexiga resultando em sinais clínicos como hematúria, polaquiúria, estrangúria e disúria, embora esses sinais possam ser observados em outras afecções do trato urinário. Os cálculos podem comprometer os mecanismos de defesa do hospedeiro, predispondo à infecção complicada do trato urinário (GRAUER, 2015). Ao exame físico os urocistólitos podem ser palpados em $20 \%$ dos felinos e cães acometidos e em alguns casos é possível sentir a parede vesical espessada (LANGSTON et al., 2008; LULICH et al., 2011; BARTGES, 2016).

Os cálculos vesicais pequenos podem alcançar a uretra e ocasionar obstrução parcial ou total, principalmente em machos, alterando o fluxo urinário, o que ocasiona distensão vesical e dor abdominal intensa, que podem ser observadas no exame físico (LANGSTON et al., 2008; GRAUER, 2015; BARTGES, 2016). O paciente também pode 
apresentar estrangúria, uremia pós-renal (anorexia, vômito e depressão) e eventual ruptura vesical, levando ao uroabdômen. A realização de palpação retal em pacientes com estrangúria e/ou disúria pode revelar presença de cálculo uretral ou distensão da uretra (em casos de obstrução) (LANGSTON et al., 2008). O gotejamento urinário é observado na maioria dos casos (PALM; WESTROPP, 2011).

Quando há presença de nefrólitos e ureterólitos, os sinais clínicos podem ser inespecíficos, intermitentes ou o paciente pode ser assintomático. Pode estar presente hematúria macroscópica, mas principalmente microscópica (KYLES et al., 2005; LULICH et al., 2011;GRAUER, 2015). A litíase renal e ureteral tem potencial de ocasionar dano no tecido dos rins e inflamação crônica, o que acarreta redução nos mecanismos de defesa do hospedeiro com aumento do risco de infecção ascendente (PIMENTA et al., 2014; GRAUER, 2015).

Cálculos localizados em ureteres também podem ocasionar obstrução parcial e/ou total podendo levar a quadros de uremia, hipercalemia e /ou acidemia (LANGSTON et al., 2008; GRAUER, 2015). Os felinos com doença renal crônica préexistente apresentam descompensação da função renal e foi observado que grande porcentagem de felinos com doença renal crônica apresentava histórico de litíase renal e/ou ureteral (GRAUER, 2015). Entretanto, não foi possível afirmar qual enfermidade desenvolveu-se primeiramente. Manifestação de dor abdominal pode estar presente, mas não é um achado comum (KYLES et al., 2005).

No exame físico de felinos sem DRC e ureterolitíase pode não haver anormalidade na palpação renal, principalmente em casos onde somente um ureter é acometido. No entanto, assimetria e dor renal podem ser observadas durante a palpação em casos obstrutivos, devido a hidronefrose (LANGSTON et al., 2008).

\section{TESTES LABORATORIAIS \\ HEMOGRAMA E PERFIL BIOQUÍMICO}

A avaliação laboratorial (hemograma e perfil bioquímico) de pacientes com urolitíase é sempre indicada, embora na maioria das vezes encontram-se normais. A análise visa verificar a presença de alguma enfermidade relacionada com o desenvolvimento de urolitíase, a qual pode ser correlacionada com a composição da pedra (BARTGES; CALLENS, 2015).

A hipercalcemia em felinos é associada a formação de urólitos de oxalato de cálcio $(\mathrm{CaOx})$ e fosfato de cálcio. Doenças que cursam com hipercalcemia devem ser investigadas nesses pacientes (LULICH; OSBORNE, 2009; PALM; WESTROPP, 2011), dessa forma é indicada a quantificação de cálcio sérico (GRAUER, 2015). A hipercalcemia idiopática foi observada em 35\% dos gatos com urólitos de oxalato de cálcio. Nos gatos, o cálcio sérico total e ionizado podem estar aumentados, entretanto a concentração de paratormônio (PTH) é geralmente baixa (PALM; WESTROPP, 2011; BARTGES, 2016).

Avaliação da função hepática é importante, visto que cálculos de urato podem ser decorrentes de disfunção nesse órgão. Em alguns casos é observada hipercalemia e acidose metabólica (ALBASAN et al., 2012; BARTGES; CALLENS, 2015). Considerando a localização do urólito, pode desenvolver-se azotemia pósrenal, em casos de obstrução em trato urinário inferior e/ou superior, de 24 à 36 horas após a obstrução (GRAUER, 2015). A litíase urinária é fator predisponente a infecções secundárias, leucocitose, em alguns casos, foi associada com pielonefrite, mas não a cistite (BARTGES, 2004). 


\section{URINÁLISE}

O exame de urina é uma parte importante no diagnóstico e terapia dos distúrbios do trato urinário, além de fornecer informações para a pesquisa de doenças em outros sistemas e órgãos. A obtenção da amostra pode ser realizada por cateterização uretral, micção natural ou cistocentese, sendo esse último o método de escolha, pelo menor grau de contaminação (LANGSTON et al., 2008; LULICH et al., 2011).

O exame de urina proveniente de pacientes com sinais clínicos compatíveis com doença urinária, normalmente demonstra alterações indicativas de um processo inflamatório, como piúria, proteinúria, hematúria e células epiteliais (OSBORNE et al., 1996). Em casos em que existe suspeita de urolitíase, o exame de urina pode auxiliar a predizer o tipo de mineral que compõe o cálculo em questão (LANGSTON et al., 2008; PALM; WESTROPP, 2011). Essa previsão ocorre por meio da densidade, $\mathrm{pH}$ urinário, cristalúria e presença ou ausência de bactérias (GRAUER, 2015).

Para isso, a análise da amostra de urina deve ser realizada no máximo até 30 minutos após a colheita, devido alterações in vitro que fornecerão resultados errôneos (ALBASAN et al., 2003). A presença de cristais na urina pode auxiliar na identificação do tipo de urólito presente no trato urinário, contudo a cristalúria é afetada pelo pH urinário (LANGSTON et al., 2008; LULICH; OSBORNE, 2009). Os urólitos de estruvita se formam em urina alcalina, fosfato de cálcio em urina alcalina para neutra, sílica e oxalato de cálcio do neutro ao ácido, urato, xantina e cistina em urina ácida (LULICH et al., 2011; GRAUER, 2015; LULICH et al., 2016).

Ainda que a cristalúria represente uma ferramenta para a identificação do mineral em questão, deve-se considerar que a presença de cristais na urina não indica que existam cálculos e nem que eles irão se formar (LULICH; OSBORNE, 2009). Também se sabe que por vezes a cristalúria pode não estar presente ou os cristais são totalmente diferentes do tipo mineral do urólito (PALM; WESTROPP, 2011).

As amostras de urina de pacientes saudáveis, que são refrigeradas ou analisadas quatro a seis horas após a coleta podem apresentar cristais de oxalato de cálcio e estruvita. Em felinos com litíase urinária a cristalúria em amostras frescas pode predizer o tipo de urólito (LANGSTON et al., 2008). Entretanto, nesses casos os cristais podem corresponder somente à camada mais externa da pedra, o que não determinaria a porção inicial do cálculo (KALINSKI et al., 2012).

Em estudo desenvolvido avaliando 46 felinos com DRC e nefrolitíase, a cristalúria foi observada apenas em seis $(13 \%)$ dos gatos, sendo cristais de oxalato de cálcio. O grupo controle era composto por 25 felinos que tinham DRC, desses, somente quatro (16\%) apresentavam cristais na urina (PIMENTA et al., 2014). Outro estudo avaliou 124 animais saudáveis e verificou cristalúria em 36 (KYLES et al., 2005).

A forma e o tempo de armazenamento das amostras influenciam na formação in vitro de cristais. Amostras refrigeradas apresentam alterações na análise química e exame do sedimento ocasionando erro na interpretação dos resultados da urinálise (ALBASAN et al., 2003; LULICH et al., 2016). Estudo comparativo entre a forma (refrigeradas e temperatura ambiente) e o tempo de armazenamento de amostras de urina de cães e gatos hígidos verificou diferença quanto a formação de cristais. A cristalúria foi mais intensa naquelas amostras submetidas à refrigeração (ALBASAN et al., 2003). 
A refrigeração das amostras é indicada para preservar as características físico-químicas da urina, ao minimizar a proliferação bacteriana in vitro. Entretanto, aumenta o grau de cristalúria, nesse estudo os principais cristais formados foram estruvita e oxalato de cálcio (ALBASAN et al., 2003; BARTGES; CALLENS, 2015).

Em alguns casos o processo de litogênese pode ser oriundo de infecção do trato urinário, principalmente por bactérias urease-positivas (Staphilococcus spp e Proteus spp) que predispõem à formação de cálculos de estruvita (LANGSTON et al., 2008; BARTGES; CALLENS, 2015). Assim, a sedimentoscopia pode revelar piúria, bacteriúria e hematúria (LANGSTON et al., 2008). Contudo, em gatos a infecção do trato urinário (ITU) normalmente é uma complicação da urolitíase, ocasionada especialmente por micro-organismos oportunistas (Streptococcus spp, Escherichia coli ) que se aproveitam das alterações no mecanismo de defesa do hospedeiro, dessa forma a cultura urinária sempre é indicada em pacientes com urólitos (OSBORNE et al., 1995; BARTGES; CALLENS, 2015; GRAUER, 2015; HECHT, 2015).

\section{EXAMES DE IMAGEM}

Os exames de imagem são considerados a ferramenta mais definitiva no diagnóstico da urolitíase (BARTGES; CALLENS, 2015; TÜRK et al., 2016). As técnicas de imagem são recomendadas, pois além de auxiliarem na identificação dos cálculos, também fornecem informações como tamanho, número, localização, formato e densidade (OSBORNE et al., 1995; RADEMACHER, 2019). A escolha do método cabe ao clínico, contudo independente da técnica utilizada a preparação do paciente para realização do exame é fundamental para melhor acurácia (ESSMAN, 2005).

A presença de gases intestinais, sombras ósseas e mau posicionamento do paciente pode obscurecer os urólitos, principalmente nefrólitos e ureterólitos (KYLES et al., 2005; LANGSTON et al., 2008; TÜRK et al., 2016). Para realização do exame, principalmente os contrastados, o paciente deve estar em jejum de 24 horas e ser realizado ao menos um enema duas horas antes da realização, pois material presente no cólon pode obscurecer a visualização da bexiga (ESSMAN, 2005). A associação de técnicas de imagem é indicada nos casos onde há forte suspeita de litíase urinária, contudo não é confirmada sua presença (GRAUER, 2015).

\section{RADIOGRAFIA SIMPLES}

A radiografia simples é um método diagnóstico importante, pois permite a identificação de urólitos, principalmente os radiopacos. Os tipos de cálculos mais facilmente observados por meio da técnica são os compostos por oxalato de cálcio e estruvita (WEICHSELBAUM et al., 1999; TÜRK et al., 2016). Todavia, o oxalato de cálcio é o mais radiopaco. Entretanto de 1,7\% a 5,2\% não aparecem nesse exame, normalmente por possuírem tamanho menor que $3 \mathrm{~mm}$ (WEICHSELBAUM et al., 1999; BARTGES; CALLENS, 2015; GRAUER, 2015).

Os cálculos de urato e cistina são mais radiolucentes e os de fosfato de cálcio possuem opacidade variável (LANGSTON et al., 2008; LULICH et al., 2016). Cerca de $25 \%$ desses urólitos não são observados na radiografia, mesmo com tamanho maior que $3 \mathrm{~mm}$ apresentando resultado falso-negativo. Os cálculos de sangue seco solidificado são radiolucentes (LULICH; OSBORNE, 2009; HOUSTON et al., 2016). A ocorrência de falso-negativo na 
radiografia para diagnóstico da doença é de 13\% (WEICHSELBAUM et al., 1999).

De maneira geral, a alteração radiográfica predominante na bexiga é a presença dos cálculos, entretanto é necessário descartar neoplasias e mineralização da parede vesical (LULICH et al., 2011; RADEMACHER, 2019). Os cálculos uretrais podem ser negligenciados, especialmente se não for incluída toda a uretra. Ao realizar o exame, é importante a inclusão da uretra pélvica e deve-se comparar as projeções laterais e dorso ventrais, para evitar erros (LANGSTON et al., 2008). A realização de projeção ventro-dorsal oblíqua minimiza a sobreposição de estruturas ósseas, que podem escurecer cálculos uretrais (HECHT, 2015).

Em pacientes com doença renal crônica (DRC) é recomendado à realização de exame radiográfico do abdômen visto que, existe um crescente número de felinos portadores da doença que apresentam nefrolitíase e urolitíase (KYLES et al., 2005). Os nefrólitos podem ser difíceis de diferenciar, pois a opacidade observada nos rins, além de cálculo renal, pode estar relacionada com calcificação do parênquima renal (nefrocalcinose), tumores calcificados, ou cistos mineralizados (GRAUER, 2015). Em seres humanos, a sensibilidade da radiografia simples para nefrólitos é de 59\% (TÜRK et al., 2016).

A opacidade observada em área ureteral geralmente indica ureterólito (LANGSTON et al., 2008). Entretanto, cálculos ureterais pequenos ou recobertos pelo conteúdo do cólon, podem ser negligenciados. A sensibilidade da radiografia no diagnóstico da ureterolitíase é de $81 \%$ (KYLES et al., 2005). A radiodensidade dos urólitos não deve ser interpretada somente como um índice de confiança da composição mineral. A avaliação radiográfica pode nos fornecer informações para predizer o tipo mineral (LANGSTON et al., 2008; LULICH; OSBORNE, 2009; PALM; WESTROPP, 2011). (Quadro 1).

QUADRO 1 - Fatores que auxiliam a predizer a composição mineral de urólitos de felinos por meio da radiografia simples, considerando as características individuais

\begin{tabular}{|c|c|c|c|c|}
\hline Mineral & $\begin{array}{c}\text { Radiopacidade } \\
\text { comparada à } \\
\text { tecido mole }\end{array}$ & $\begin{array}{l}\text { Contorno de } \\
\text { superfície }\end{array}$ & Forma & $\begin{array}{l}\text { Tamanho } \\
\text { aproximado }\end{array}$ \\
\hline $\begin{array}{l}\mathrm{CaOx} \\
\text { monohidratado }\end{array}$ & $+++\mathrm{a}++++$ & $\begin{array}{l}\text { Liso, } \\
\text { ocasionalmente } \\
\text { com projeções }\end{array}$ & $\begin{array}{l}\text { Geralmente } \\
\text { Redondo, mas } \\
\text { por vezes em } \\
\text { forma de } \\
\text { roseta }\end{array}$ & $1 \mathrm{~mm}-5 \mathrm{~mm}$ \\
\hline $\begin{array}{l}\mathrm{CaOx} \\
\text { diidratado }\end{array}$ & $+++\mathrm{a}++++$ & Rugosa a lisa & Roseta & $1 \mathrm{~mm}-7 \mathrm{~mm}$ \\
\hline Estruvita estéril & $++\mathrm{a}+++$ & $\begin{array}{l}\text { Levemente } \\
\text { rugoso }\end{array}$ & $\begin{array}{l}\text { Redondo a } \\
\text { discóide }\end{array}$ & $3 m m-10 m m$ \\
\hline $\begin{array}{l}\text { Estruvita por } \\
\text { infecção }\end{array}$ & $+a+++$ & $\begin{array}{ll}\text { Liso } & \text { a } \\
\text { levemente } & \\
\text { rugoso } & \end{array}$ & $\begin{array}{l}\text { Redondo } \\
\text { facetada }\end{array}$ & $2 \mathrm{~mm}->7 \mathrm{~mm}$ \\
\hline
\end{tabular}




\begin{tabular}{|l|l|l|l|l|}
\hline Urato & $-\mathrm{a}++$ & Liso & $\begin{array}{l}\text { Redondo a } \\
\text { ovóide }\end{array}$ & $2 \mathrm{~mm}-10 \mathrm{~mm}$ \\
\hline $\begin{array}{l}\text { Fosfato de } \\
\text { cálcio }\end{array}$ & $+++\mathrm{a}++++$ & Rugosa & $\begin{array}{l}\text { Rara } \\
\text { ocorrência }\end{array}$ & $1 \mathrm{~mm}-4 \mathrm{~mm}$ \\
\hline Cistina & $-\mathrm{a}+++$ & Rugosa & Redondo & $1 \mathrm{~mm}-4 \mathrm{~mm}$ \\
\hline Sílica & $++\mathrm{a}++++$ & Rara ocorrência & $\begin{array}{l}\text { Rara } \\
\text { ocorrência }\end{array}$ & $1 \mathrm{~mm}-4 \mathrm{~mm}$ \\
\hline Xantina & $-\mathrm{a}+$ & Liso & $\begin{array}{l}\text { Redondo a } \\
\text { ovóide }\end{array}$ & $1 \mathrm{~mm}-5 \mathrm{~mm}$ \\
\hline
\end{tabular}

Fonte: Adaptado de Lulich et al. (2011).

\section{RADIOGRAFIA CONTRASTADA}

A técnica contrastada é indicada nos casos onde existe a suspeita de urolitíase, entretanto não é observado no exame simples. O método proporciona maior sensibilidade para detecção de cálculos pequenos e/ou radiolucentes, além de melhorar o diagnóstico de urocistólitos (BARTGES; CALLENS, 2015; GRAUER, 2015; HECHT, 2015). As técnicas radiográficas contrastadas para diagnóstico da urolitíase consistem em cistografia de contraste negativo, contraste positivo, contraste duplo e urografia excretora (HECHT, 2015; RADEMACHER, 2019).

A radiografia de contraste negativo (cistografia de contraste negativo) é mais sensível no diagnóstico de urolitíase vesical quando comparada com a radiografia simples, todavia menos efetiva que a de contraste duplo, com falsonegativo de 6,5\% (WEICHSELBAUM et al., 1999). A técnica consiste na utilização de um agente gasoso como contraste, o qual pode ser o ar ambiente, dióxido de carbono ou óxido nitroso. (ESSMAN, 2005; HECHT, 2015).

O dióxido de carbono e o óxido nitroso são 20 vezes mais solúveis no sangue que o ar ambiente, desse modo o risco de embolia gasoso fatal é menor. $O$ urólito pode ser maior do que aparenta na radiografia, se apenas uma porção contem minerais radiodensos. Dentre as vantagens da técnica estão a facilidade de execução e custo baixo, contudo cálculos pequenos podem ser negligenciados (OSBORNE et al., 1996; ESSMAN, 2005).

O método de contraste positivo consiste na aplicação de um contraste iodado. $\mathrm{O}$ volume de contraste utilizado é cerca de $10 \mathrm{~mL} / \mathrm{kg}$ diluído em solução fisiológica ou água estéril a $10 \%$ a $20 \%$. A aplicação é lenta e cessa ao sentir a bexiga túrgida à palpação (ESSMAN, 2005; HECHT, 2015).

As pedras que aparecem radiodensas na radiografia simples podem apresentar-se radiolucentes na radiografia de contraste positivo (OSBORNE et al., 1996; ESSMAN, 2005). A justificativa está relacionada com o fato de que são mais radiodensas que o tecido, no entanto menos que o contraste. O diagnóstico de urólito radiolucente deve ser considerado comparando-se a radiodensidade com tecidos moles e não com o material de contraste. A técnica é mais indicada para avaliação da integridade vesical, pois pode atrapalhar a visualização dos urólitos (OSBORNE et al., 1996; LANGSTON et al., 2008; LULICH et al., 2011).

A radiografia com contraste duplo é a mais precisa dentre as técnicas contrastadas, com falso negativo de $4,5 \%$. O método consiste em distender a bexiga com gás e em seguida proceder à administração de contraste iodado (aproximadamente 1 a $3 \mathrm{~mL}$ ) (WEICHSELBAUM et al., 1999). $\mathrm{Na}$ cistografia de duplo contraste, os cálculos tendem a permanecer no centro do contraste e podem apresentar superfície lisa ou irregular. 
Bolhas de ar podem aparecer iatrogenicamente na cistografia e ser confundidas com cálculos, entretanto estas tendem a permanecer na periferia do contraste e são perfeitamente redondas e com bordas lisas (ESSMAN, 2005). Embora seja um excelente método para o diagnóstico a acurácia é de $53 \%$ dos casos (WEICHSELBAUM et al., 1999).

A urografia excretora (UE) auxilia no diagnóstico de cálculos em trato urinário superior (LANGSTON et al., 2008). Em medicina humana, foi considerada durante vários anos como o melhor exame de imagem para abordagem da urolitíase (TÜRK et al., 2016).Nessa técnica é aplicado contraste iodado pela via intravenosa (IV) e procede-se com realização de radiografias imediatamente à aplicação do contraste e em cinco, 20 e 40 minutos após (ESSMAN, 2005). Contudo, tanto na medicina quanto na veterinária, áreas com falta do contraste, podem estar relacionadas com cistos, neoplasias, granulomas, abscessos, hematomas e não somente com cálculos (LANGSTON et al., 2008).

Atualmente o exame é mais indicado para visualização do sistema coletor urinário, embora seja possível a identificação de nefrólitos, ureterólitos e obstrução ureteral (BARTGES; CALLENS, 2015; RADEMACHER, 2019). Estudos realizados em pacientes humanos relatam que a UE é sensível apenas em $52 \%$ a $69 \%$ na detecção das pedras (TÜRK et al., 2016). O contraste pode interferir com o resultado do exame de urina, ocasionando aumento da densidade e proteína, portanto recomenda-se a realização de urocultura e/ou urinálise somente 24 horas após a urografia contrastada (LANGSTON et al., 2008).

Em pacientes desidratados, anúricos ou que tenham hipersensibilidade ao contraste iodado a urografia excretora é contra indicada (ESSMAN, 2005). Nos casos em que não se visualizam cálculos no exame radiográfico simples, opta-se primeiramente pela ultrassonografia abdominal aos exames contrastados (HECHT, 2015).

\section{ULTRASSONOGRAFIA ABDOMINAL}

A ultrassonografia abdominal é indicada para detecção de urólitos radiolucentes, em casos de uropatia obstrutiva resultando em dilatação ureteral ou de pelve renal e em felinos com azotemia (PALM; WESTROPP, 2011; GRAUER, 2015). Embora considerada melhor para o diagnóstico da litíase urinária quando comparada com a radiografia simples, a taxa de falso-negativo é similar à radiografia de duplo-contraste com 3,5\% (LANGSTON et al., 2008).

O uso de transdutor $7.5 \mathrm{MHz}$ aumenta a sensibilidade na detecção de urocistólitos, entretanto caso seja utilizado um transdutor $5.0 \mathrm{MHz}$ ou de menor frequência, resultados falso-negativos são comuns (WEICHSELBAUM et al., 1999; TÜRK et al., 2016). A técnica de ultrassonografia abdominal tem a vantagem de não expor o paciente e o técnico que realiza o exame à radiação, além de custo reduzido associado a melhor qualidade de imagem. Entretanto, o exame não é recomendado para predizer a composição mineral do urólito (LULICH; OSBORNE, 2009).

A maioria dos urólitos vesicais independentemente de serem radiopacos ou não, tendem a aparecer na ultrassonografia. A interface entre a urina e os cálculos é intensamente hiperecóica, com sombras acústicas entre os cálculos. A visualização de uretrólitos somente é possível se estiverem próximo à bexiga, uma vez que não é possível a observação da uretra pélvica nesse exame, portanto cálculos nessa região podem ser negligenciados (LANGSTON et al., 2008). 
Os urólitos muito pequenos podem não ser diagnosticados por não produzirem sombra acústica ou serem confundidos com depósito de cristais (LANGSTON et al., 2008; BARTGES; CALLENS, 2015). A dilatação da pelve renal ou ureteral é facilmente detectada indicando obstrução ureteral parcial ou total, o que sugere presença de ureterólito (LANGSTON et al., 2008; LULICH et al., 2011). Estudo em felinos a US foi mais sensível na detecção de cálculos ureterais, apresentando taxa de $77 \%$, quando comparada com a radiografia simples, chegando a 90\% quando associada a técnicas radiográficas (KYLES et al., 2005).

\section{TOMOGRAFIA COMPUTADORIZADA}

A tomografia computadorizada (TC) têm se popularizado como melhor método no diagnóstico da litíase urinária em medicina, uma vez que apresenta 94\% de sensibilidade na detecção dos cálculos, além de fornecer alternativas diagnósticas para dor lombar aguda (TÜRK et al., 2016; NYKAMP, 2017). Essa técnica permite facilmente a distinção de urólitos dos tecidos moles circundantes (PRESSLER et al., 2004). Em estudo realizado com felinos, a tomografia computadorizada foi capaz de diagnosticar cálculo ureteral em três pacientes, cujo urólitos não haviam sido observados na radiografia simples e ultrassonografia abdominal (KYLES et al., 2005).

Além da capacidade de detecção da presença de urólitos, outra aplicação do exame seria a possibilidade de predizer a composição dos cálculos com base nas diferentes densidades (PRESSLER et al., 2004; NYKAMP, 2017). A determinação da composição in vivo beneficia o tratamento médico ao facilitar a escolha terapêutica, quando não há possibilidade de remoção da pedra. Estudo in vitro com cálculos removidos de cães demonstrou ter $75 \%$ a $88 \%$ de acurácia para urólitos puros, não sendo confiável em cálculos mistos (PRESSLER et al., 2004).

Estudo desenvolvido in vitro com urólitos de pacientes humanos utilizando tomografia computadorizada não-contrastada foi capaz de determinar a composição por meio da densidade de cada amostra à $120 \mathrm{kV}$. Os cálculos identificados do menos ao mais denso foram ácido úrico, estruvita, cistina, fosfato de cálcio e oxalato de cálcio monohidratado, Também identificaram-se diferentes composições na mesma amostra. A utilização de energia dupla a $80 \mathrm{kV}$ e $120 \mathrm{kV}$ não apresentou diferença significativa para a análise da composição de urólitos humanos (DEVECI et al., 2004).

A tomografia computadorizada tem a vantagem de ser um método rápido de realização e a análise não é invasiva (PRESSLER et al., 2004). Entretanto, a avaliação da densidade para predizer a composição do urólito é subjetiva, visto que pode haver variabilidade entre os diferentes analistas e diferentes tomógrafos (NYKAMP, 2017). Em estudo a utilização desse método apresentou acurácia de $64 \%$ a $81 \%$. Na realização do exame pode haver erros devido ao tamanho do paciente o que influencia a sobreposição de diferentes composições no cálculo. A tomografia, entretanto, é um método relativamente caro e de difícil acesso, principalmente em medicina veterinária (PRESSLER et al., 2004; ZHANG et al., 2016).

\section{ANÁLISE DA COMPOSIÇÃO DO URÓLITO}

Os exames laboratoriais e de imagem contribuem no diagnóstico da doença e podem indicar o tipo de urolitíase, contudo nenhum desses procedimentos é extremamente confiável devido às variações na forma, tamanho e artefatos de imagem (PRESSLER et 
al., 2004; ZHANG et al., 2016). O diagnóstico definitivo ocorre somente com a investigação da composição do cálculo (LANGSTON et al., 2008; GRAUER, 2015).

Essa determinação é significativa em termos de terapia e prevenção e é considerada parte integrante da avaliação de pacientes com litíase urinária, visto que auxilia no entendimento do desenvolvimento da doença (KALINSKI et al., 2012; BLASCHKO et al., 2013; SIENER et al., 2016). A análise é realizada com o uso de técnicas qualitativas e quantitativas, qualquer falha na detecção das substâncias pode resultar em terapia incorreta. A pedra pode ser obtida pela micção natural ou remoção cirúrgica. Em casos que o paciente apresente mais de um urólito deve-se proceder com a análise de todos (GRAUER, 2015; SIENER et al., 2016).

\section{ANÁLISE QUALITATIVA QUÍMICA}

A análise qualitativa química é uma técnica realizada por diversos laboratórios, entretanto sua confiabilidade é "variável". O método consiste na pulverização da amostra e o pó é submetido a reagentes específicos que produzem mudanças de cor na presença de certos componentes (cálcio, magnésio, ácido úrico, cistina, amônio) (RUBY; LING, 1986; KOEHLER et al., 2009; CLOUTIER et al., 2015).

Como a amostra é pulverizada não é possível diferenciar as camadas e nem a presença de diferentes constituintes por camada (CLOUTIER et al., 2015). Também não se consegue quantificar o percentual dos minerais presentes, sendo impossível classificar a amostra como simples, mista ou composta (ULRICH et al., 1996; LULICH et al., 2011; CLOUTIER et al., 2015).

Os reagentes utilizados nesse tipo de análise não detectam as substâncias em quantidades menores que 20\% (RUBY; LING, 1986). Componentes como a sílica e xantina, cistina, fármacos também não são detectados (ULRICH et al., 1996; KOEHLER et al., 2009; LULICH; OSBORNE, 2009). A técnica é pouco sensível na detecção de cálcio podendo apresentar falso-positivos. Em casos de oxalato pode-se observar resultados falso-negativos. Portanto, os falso-negativos e falso-positivos são comuns nesses testes (RUBY; LING, 1986; LULICH; OSBORNE, 2009). Conforme descrito por Hesse et al. (2005) com urólitos de pacientes humanos, a análise química apresentou percentual de erro de $94 \%$, enquanto Siener et al. (2016) a taxa de erro foi de $90 \%$.

\section{ANÁLISE FÍSICA QUANTITATIVA}

A análise física quantitativa é a mais indicada, pois permite identificar as camadas do urólito, determinar e quantificar as substâncias presentes em cada porção, além de diferenciar subdivisões nos grupos minerais (oxalato de cálcio monohidratado e diidratado). Também permite a classificação do cálculo em simples, misto e composto (RUBY; LING, 1986; LANGSTON et al., 2008).

As técnicas físicas quantitativas disponíveis são a microscopia de luz polarizada, difração de raios- $x$, espectroscopia infravermelha e espectroscopia de energia dispersiva (RUBY; LING, 1986; ULRICH et al., 1996; LULICH; OSBORNE, 2009).

\section{MICROSCOPIA DE LUZ POLARIZADA}

A microscopia de luz polarizada (MLP) baseia-se na interação da luz polarizada com os cristais por meio de um microscópio polarizador, que identifica os componentes cristalinos dos urólitos. As partes representativas são identificadas usando um microscópio de 
dissecação (ULRICH et al., 1996). As propriedades ópticas, como a cor, índice de refração e birrefringência são parâmetros comparados com padrões conhecidos e servem para determinar a composição da pedra (SCHUBERT, 2006; LULICH; OSBORNE, 2009).

Como vantagens da técnica estão custo favorável, rapidez na realização do exame e possibilidade de análise de amostras pequenas. Também permite a diferenciação do grau de hidratação dos urólitos de oxalato de cálcio (diidratado e monohidratado) e detecção de componentes presentes em quantidade mínima. Entre as desvantagens cita-se dificuldade da análise quantitativa em urólitos mistos e impossibilidade de diferenciação dos cálculos derivados de purina (como urato e xantina) (SCHUBERT, 2006).

\section{DIFRAÇÃO DE RAIOS-X}

A difração de raios-x é o método preferido para identificação da composição de urólitos, pois possui alto grau de confiabilidade. A técnica consiste na difração de raios- $x$ na estrutura cristalina da amostra. $O$ material cristalino ao receber os raios-x emite uma "impressão digital" (padrão único de difração), o qual é registrado permitindo comparação com padrões de referência conhecidos e identificação das substâncias (ULRICH et al., 1996; BLASCHKO et al., 2013; SIENER et al., 2016).

As vantagens desse método incluem fácil preparo, medição automática dos componentes, bem como troca automática de amostra, avaliação semiautomática do difratograma de raios-x (busca automática de correspondência com padrão) e diferenciação exata de todos os componentes cristalinos (SCHUBERT, 2006). Como desvantagens observa-se alto custo, detecção somente de componentes cristalinos e o tempo para avaliação de uma amostra é de 30 minutos. A presença de mais de três componentes no urólito reduz a eficácia do método. (SCHUBERT, 2006; BLASCHKO et al., 2013).

Em estudo comparando a análise química, espectroscopia infravermelha e difração de raios-x, essa útlima apresentou maior acurácia na identificação dos tipos de urolitíase, bem como os subtipos dos minerais os urólitos de pacientes humanos (SIENER et al., 2016).

\section{ESPECTROSCOPIA INFRAVERMELHA}

Esse método envolve o uso do espectrofotômetro que possui um espectro de luz infravermelha com ondas. O equipamento expõe as moléculas dos componentes da amostra à luz infravermelha ocasionando interação. A luz simula vibrações atômicas e como consequência tem-se absorção de energia que resultam em ondas de absorção (RUBY; LING, 1986; ULRICH et al., 1996; SCHUBERT, 2006).

Quando as ondas infravermelhas encontram uma amostra, algumas são absorvidas (absorvância) e outras atravessam a amostra (transmissão) formando um espectro. O espectro resultante é uma "impressão digital" molecular da amostra, como nenhuma estrutura molecular produz o mesmo espectro infravermelho, esses são comparados aos espectros de referência (ULRICH et al., 1996; LULICH; OSBORNE, 2009).

Este procedimento é útil na identificação de minerais desconhecidos, determinando a qualidade e consistência das amostras e quantificando as diferentes substâncias calculogênicas que compoem a pedra. A espectroscopia infravermelha (El) é indicada 
em casos onde o material cristalino não pode ser identificado somente pela microscopia de luz polarizada (ULRICH et al., 1996; LULICH; OSBORNE, 2009).

A espectroscopia infravermelha transformada de Fourier (FT-IR) é um tipo de técnica que mede a absorção ou emissão de luz infravermelha a partir de uma amostra (CLOUTIER et al., 2015). Os modos vibracional e rotacional de átomos em uma molécula são excitados por radiação infravermelha. A absorção ou emissão infravermelha pode ser usada para estudar a natureza molecular dos títulos. As substâncias possuem padrão e grau de absorvência diferentes e a absorção é proporcional a concentração (BLASCHKO et al., 2013).

A vantagem da EI-FT comparada ao método tradicional é que esta avalia simultaneamente todas as frequências no espectro infravermelho, utilizando um espelho móvel e fixo no interferômetro que acelera a análise da amostra. Na maioria dos laboratórios humanos é a principal técnica analítica utilizada (BLASCHKO et al., 2013).

As vantagens do uso desse método incluem custo moderado, análise rápida quando utilizada a El-FT, avaliação de amostras pequenas e detecção de substâncias não cristalinas como proteínas e gordura. O custo é menor quando comparado com difração de raios-x. Contudo, as desvantagens incluem a demora na preparação da amostra, dificuldade de diferenciação de urólitos de purinas e dificuldade para diferenciar subtipos como oxalato de cálcio diidratado e monohidratado (SCHUBERT, 2006). Estudo desenvolvido com urólitos de pacientes humanos demonstrou taxa de erro de 33\% (SIENER et al., 2016).

\section{ESPECTROSCOPIA DE ENERGIA DISPERSIVA}

A técnica consiste na dispersão de eletróns e é realizada com o auxilio de um microscópio eletrônico de varredura (MEV). O material cristalino em questão é localizado na tela do scanner e um padrão elementar é gravado (ULRICH et al., 1996).

Em estudo desenvolvido em pacientes humanos, pesquisadores investigaram a composição de nove nefrólitos por meio do EDS. Para urólitos compostos de ácido úrico, o EDS demonstrou picos de carbono, oxigênio e nitrogênio. Enquanto urólitos de oxalato de cálcio, apresentaram picos de cálcio, carbono e oxigênio, ao passo que em cálculos de estruvita foi observado picos de magnésio, fósforo, oxigênio, carbono e nitrogênio (LEE et al., 2012).

O EDS permite a avaliação das diferentes regiões da pedra, determinação da análise elementar e composição química em cada região. Fornece análise qualitativa rápida e simples, o que facilita o correto diagnóstico e tratamento pelos clínicos. Também pode ser utilizado quando se deseja a comparação em uma amostra, em casos de urólitos de oxalato de cálcio monohidratado e diidratado (LEE et al., 2012).

O EDS permite a gravação e arquivamento da "quantidade" de radiação criada como resultado da excitação de elétrons secundários da superfície das amostras analisadas. Isso permite a composição elementar de matéria orgânica e inorgânica (KALINSKI et al., 2012).

A espectroscopia de energia dispersiva é um método que permite resultados rápidos, a amostra não é destruída, sendo possível repetir a análise e submeter a outros estudos(KALINSKI et al., 2012). Estudo desenvolvido por Shamema et al. (2015) o EDS detectou zircônio e mercúrio em cálculos urinários de pacientes humanos, substâncias que não 
haviam sido identificadas por meio de difração de raios-x e FT-IR, o que melhorou a confiabilidade do resultado.

Essa é uma técnica eficaz para determinação de compostos inorgânicos, entretanto não diferencia os tipos de fosfato de cálcio (brushita e apatita) e materiais orgânicos como urato e xantina, nesses casos recomenda-se 0 uso da espectroscopia infravermelha (RUBY; LING, 1986).

Todas as técnicas de análise possuem vantagens e desvantagens, contudo nenhum método é suficiente para fornecer totais informações sobre a composição das pedras, dessa forma recomenda-se a associação das técnicas, o que potencializa a confiabilidade do resultado (SHAMEMA et al., 2015).

\section{CONSIDERAÇÕES FINAIS}

Embora os sinais clínicos apresentados por pacientes com urolitíase sejam bastante inespecíficos, ou até mesmo ausentes, o diagnóstico da enfermidade não é desafiador. Entretanto, o fator mais importante para a instituição de tratamento e medidas preventivas corretas é a determinação da composição do urólito. Dessa forma, a remoção do cálculo, na maioria das vezes, não representa a solução do problema.

Os métodos de diagnóstico não devem ser considerados isoladamente para determinar a composição da pedra, visto que, estão sujeitos a erros de interpretação o que ocasiona falha na terapia, podendo agravar o problema. Apesar das pesquisas envolvendo o uso da tomografia computadorizada não contrastada para predizer as substâncias presentes nos cálculos, este ainda é um exame que necessita de mais investigações, além de até o momento não ter sido capaz de identificar todos os componentes da amostra. Sendo assim, as técnicas de análise da composição são as melhores alternativas e as formas quantitativas sobrepõemse as qualitativas.

A escolha da técnica baseia-se na disponibilidade, custo, rapidez e confiabilidade do resultado e muitas vezes a associação entre métodos é indicada. A determinação é tomada pelo clínico responsável, pois o sucesso no tratamento depende dessa análise. Em casos onde não exista possibilidade da análise quantitativa pode optar-se pela qualitativa, contudo não deve ser a primeira escolha.

A urolitíase deve sempre ser considerada em felinos com doença em trato urinário, pois é a segunda maior causa de alteração nesse sistema. Além de apresentar crescente número em sua incidência. A solicitação de exames de imagem deve ser realizada em qualquer paciente com sinais clínicos e histórico anterior da doença, evitando que recidivas sejam negligenciadas. A litíase urinária é uma importante doença que não envolve somente o trato urinário, portanto maior atenção às causas de sua ocorrência é necessária.

\section{REFERÊNCIAS}

ALBASAN, H.; LULICH, J.P.; OSBORNE, C.A.; LEKCHAROENSUK, C.; ULRICH, L.K.; CARPENTER, K.A. Effects of storage time and temperature on $\mathrm{pH}$, specific gravity, and crystal formation in urine samples from dogs and cats. Journal of the American Veterinary Medical Association, v. 222, n. 2, p. 176-179, 2003. Disponível em: <https://doi.org/10.2460/javma.2003.222.176>. doi: org/10.2460/javma.2003.222.176

ALBASAN, H.; OSBORNE, C.; LULICH, J.; LEKCHAROENSUK, C. Risk factors for urate uroliths in cats. Journal of the American 
Veterinary Medical Association, v. 240, n.7, p. 842-847, 2012. Disponível em: <https://doi.org/10.2460/javma.240.7.842>. doi: org/10.2460/javma.240.7.842

BARTGES, J.W. Diagnosis of urinary tract infections. Veterinary Clinics of North America: Small Animal Practice, v. 34, n.4, p. 923-933, 2004. Disponível em: <https://doi.org/10.1016/j.cvsm.2004.03.001 >. doi: org/10.1016/j.cvsm.2004.03.001

BARTGES, J.W.; CALLENS, A.J. Urolithiasis. Veterinary Clinics of North America: Small Animal Practice, v. 45, n. 4, p. 747-768, 2015. Disponível em: < https://doi.org/10.1016/j.cvsm.2015.03.001>. doi: org/10.1016/j.cvsm.2015.03.001

BARTGES, J.W. Feline Calcium oxalate urolithiasis: Risk factors and rational treatment approaches. Journal of Feline Medicine and Surgery, v. 18, n. 9, p. 712722, 2016. Disponível em: <https://doi.org/10.1177/1098612X16660442>. doi: $10.1177 / 1098612 \times 16660442$

BLASCHKO S.D.; MILLER, J.; CHI, T.; FLECHNER, L.; FAKRA, S.; KAHN, A.; KAPAHI, P.; STOLLER, M.L. Microcomposition of human urinary calculi using advanced imaging techniques. The Journal of Urology, v. 189, n. 2, p. 726-734, 2013. Disponível em: <https://www.ncbi.nlm.nih.gov/pmc/articles/PMC4124914/>. doi: 10.1016/j.juro.2012.09.098

CANNON, A.B.; WESTROPP, J.L.; RUBY, A.L.; KASS, P.H. Evaluation of trends in urolith composition in cats: 5,230 cases (1985-2004). Journal of the American Veterinary Medical Association, v. 231, n. 4, p. 570-576, 2007. doi: Disponível em: <https://doi.org/10.2460/javma.231.4.570>. doi: org/10.2460/javma.231.4.570

CLOUTIER, J.; VILLA, L.; TRAXER, O.; DAUDON, M. Kidney stone analysis: "Give me your stone, I will tell you who you are!". World Journal of Urology, v. 33, n. 2, p. 157-169, 2015.2 Disponível em: <https://www.ncbi.nlm.nih.gov/pmc/articles/PMC4308647/>. doi: 10.1007/s00345014-1444-9

DEVECI, S.; COŞKUN, M.; TEKIN, M.I.; PEŞKIRCIOGLU, L.; TARHAN, N.C.; OZKARDEŞ, $H$. Spiral computed tomography: role in determination of chemical compositions of pure and mixed urinary stones--an in vitro study. Urology, v. 64, n. 2, p. 237-240, 2004. Disponível em: <https://doi.org/10.1016/j.urology.2004.03.029>. doi: 10.1016/j.urology.2004.03.029

ESSMAN, S.C. Contrast cystography. Clinical Techniques in Small Animal Practice, v. 20, n. 1, p. 46-51, 2005. Disponível em: <https://www.ncbi.nlm.nih.gov/pubmed/15822530>. doi: 10.1053/j.ctsap.2004.12.007

GOMES, V.D.R.; ARIZA, P.C.; BORGES, N.C.; SCHULZ JUNIOR, F.J.; FIORAVANTI, M.C.S. Risk factors associated with feline urolithiasis. Veterinary Research Communications, v. 42, n. 1, p. 87-94, 2018. Disponível em: <https://www.ncbi.nlm.nih.gov/pubmed/29340849>. doi: 10.1007/s11259-018-9710-8

GRAUER, G.F. Feline Struvite \& Calcium Oxalate Urolithiasis.

Today's Veterinary Practice, v. 5, n. 5, p.14-20, 2015. Disponível 
em: <https://todaysveterinarypractice.com/feline-struvite-calcium-oxalateurolithiasis/>.

HECHT, S. Diagnostic imaging of lower urinary tract disease. Veterinary Clinics of North America: Small Animal Practice, v. 45, n. 4, p. 639-663, 2015. Disponível em: <https://doi.org/10.1016/j.cvsm.2015.02.002>. doi: 10.1016/j.cvsm.2015.02.002

HESSE, A.; KRUSE, R.; GEILENKEUSER, W.J.; SCHMIDT, M. Quality control in urinary stone analysis: results of 44 ring trials (1980-2001). Clinical Chemistry and Laboratory Medicine, v. 43, n. 3, p. 298-303, 2005. Disponível em: <https://doi.org/10.1515/CCLM.2005.051 > . doi: 10.1515/CCLM.2005.051

HOUSTON, D.M.; MOORE, A.E. Canine and feline urolithiasis: examination of over 50000 urolith submissions to the Canadian veterinary urolith centre from 1998 to 2008. The Canadian Veterinary Journal, v. 50, n. 12, p.1263-1268, 2009. Disponível em: < https://www.ncbi.nlm.nih.gov/pmc/articles/PMC2777289/>.

HOUSTON, D.M.; VANSTONE, N.P.; MOORE, A.E.; WEESE, H.E. Evaluation of 21426 feline bladder urolith submissions to the Canadian Veterinary Urolith Centre (1998-2014). The Canadian Veterinary Journal, v. 57, n. 2, p. 196-201, 2016. Disponível em: < https://www.ncbi.nlm.nih.gov/pmc/articles/PMC4713001/ /> .

KALINSKI, K.; MARYCZ, K.; CZOGALA, J.; SERWA, E.; JANECZEK, W. An application of scanning electron microscopy combined with roentgen microanalysis (SEM-EDS) in canine urolithiasis. Journal of Electron Microscopy, v. 61, n. 1, p. 47-55, 2012. Disponível em: <https://doi.org/10.1093/jmicro/dfr086>. doi: 10.1093/jmicro/dfr086

KOEHLER, L.A.; OSBORNE, C.A.; BUETTNER, M.T.; LULICH, J.P.; BEHNKE, R. Canine uroliths: frequently asked questions and their answers. Veterinary Clinics of North America: Small Animal Practice, v. 39, n. 1, p.161-181, 2009. Disponível em: < https://doi.org/10.1016/j.cvsm.2008.09.007>. doi: 10.1016/j.cvsm.2008.09.007

KYLES, A.E.; HARDIE, E.M.; WOODEN, B.G.; ADIN, C.A.; STONE, E.A.; GREGORY, C.R., et al. Clinical, clinicopathologic, radiographic, and ultrasonographic abnormalities in cats with ureteral calculi: 163 cases (1984-2002). Journal of the American Veterinary Medical Association, v. 226, n. 6, p. 932-936, 2005. Disponível em: <https://doi.org/10.2460/javma.2005.226.932>. doi: 10.2460/javma.2005.226.932

LANGSTON, C.; GISSELMAN, K.; PALMA, D.; MCCUE, J. Diagnosis of urolithiasis. Compendium on Continuing Education for the Practising Veterinarian, v. 30, n. 8, p. 447-450, 2008.2 Disponível em: <https://www.researchgate.net/publication/23298107_Diagnosis_of_urolithiasis>.

LEE, H.P.; LEONG, D.; HENG, C.T. Characterization of kidney stones using thermogravimetric analysis with electron dispersive spectroscopy. Urological Research, v. 40, n. 3, p. 197-204, 2012. Disponível em: < https://link.springer.com/article/10.1007\%2Fs00240-011-0428-0>. doi: 10.1007/s00240-011-0428-0 
LULICH, J.P.; OSBORNE, C.A. Changing paradigms in the diagnosis of urolithiasis. Veterinary Clinics of North America: Small Animal Practice, v. 39, n. 1, p. 79-91, 2009. Disponível em: <https://doi.org/10.1016/j.cvsm.2008.10.005> . doi: 10.1016/j.cvsm.2008.10.005

LULICH, J.P.; OSBORNE, C.A.; ALBASAN, H. Canine and feline urolithiasis: diagnosis, treatment, and prevention. In: Bartges, J.; Polzin, D.J. Nephrology and urology of small animals. West Sussex: Wiley-Blackwell; 2011. p.687-706.

LULICH, J.P.; BERENT, A.C.; ADAMS, L.G.; WESTROPP, J.L.; BARTGES, J.W.; OSBORNE, C.A. ACVIM small animal consensus recommendations on the treatment and prevention of uroliths in dogs and cats. Journal of Veterinary Internal Medicine, v. 30, n. 5, p. 1564-1574, 2016. Disponível em: <https://www.ncbi.nlm.nih.gov/pmc/articles/PMC5032870/>. doi: 10.1111/jvim.14559

NYKAMP, S.G. Dual-energy computed tomography of canine uroliths. American Journal of Veterinary Research, v. 2017, n.10, p. 1150-1155, 2017. Disponível em: <https://doi.org/10.2460/ajvr.78.10.1150>. doi: 10.2460/ajvr.78.10.1150

OSBORNE, C.A.; LULICH, J.P.; BARTGES, J.W.; UNGER, L.K.; THUMCHAI, R.; KOEHLER, L.A.; et al. Canine and Feline Urolithiases: Relationship of Etiopathogenesis to Treatment and Prevention. In: OSBORNE, C.A.; FINCO, D.R. Canine and Feline Nephrology and Urology, Baltimore: Williams \& Wilkins; 1995. p. $798-888$.

OSBORNE CA, LULICH JP, THUMCHAI R, BARTGES JW, SANDERSON SL, ULRICH LK, KOEHLER LA, BIRD KA, SWANSON LL. Diagnosis, medical treatment, and prognosis of feline urolithiasis. Veterinary Clinics of North America: Small Animal Practice, v. 26, n. 3, p. 589-627, 1996. Disponível em: <https://doi.org/10.1016/S0195-5616(96)50087-2>. doi: 10.1016/S01955616(96)50087-2

PALM, C,; WESTROPP, J. Cats and calcium oxalate: strategies for managing lower and upper tract stone disease. Journal of Feline Medicine Surgery, v. 13, n. 9, p. 651-660, 2011. Disponível em: <https://doi.org/10.1016/j.fms.2011.07.018> . doi: 10.1016/j.jfms.2011.07.018

PIMENTA, M.M.; RECHE-JÚNIOR, A.; FREITAS, M.F.; KOGIKA, M.M.; HAGIWARA, M.K. Estudo da ocorrência de litíase renal e ureteral em gatos com doença renal crônica. Pesquisa Veterinária Brasileira, v. 34, n. 6, p. 555-561, 2014. Disponível em: $\quad<h t t p: / / d x . d o i . o r g / 10.1590 / S 0100-736 X 2014000600009>$. doi: http://dx.doi.org/10.1590/S0100-736X2014000600009

PINEDA, C.; AGUILERA-TEJERO, E.; RAYA, A.I.; MONTES, O.A.; RODRIGUEZ, M.; LOPEZ, I. Effects of two calculolytic diets on parameters of feline mineral metabolism. Journal of Small Animal Practice, v. 56, n. 8, p. 499-504, 2015. Disponível em: < https://onlinelibrary.wiley.com/doi/full/10.1111/jsap.12368>. doi: 10.1111/jsap. 12368 
PRESSLER, B.M.; MOHAMMADIAN, L.A.; LI, E.; VADEN, S.L.; LEVINE, J.F.; MATHEWS, K.G.; ROBERTSON, I.D. In vitro prediction of canine urolith mineral composition using computed tomographic mean beam attenuation measurements. Veterinary Radiology \& Ultrasound, v. 45, n. 3, p. 189-197, 2004. Disponível em: <https://doi.org/10.1111/j.1740-8261.2004.04032.x> . doi: 10.1111/j.17408261.2004.04032.x

RADEMACHER, N. Diagnostic Imaging of the urinary tract. Veterinary Clinics of North America: Small Animal Practice, v. 49, n. 2, p. 261-286, 2019. Disponível em: <https://doi.org/10.1016/j.cvsm.2018.10.006>. doi: 10.1016/j.cvsm.2018.10.006

RUBY, A.L.; LING, G.V. Methods of analysis of canine uroliths. Veterinary Clinics of North America: Small Animal Practice, v. 16, n. 2, p. 293-301, 1986. Disponível em: <https://doi.org/10.1016/S0195-5616(86)50031-0>. doi: 10.1016/S01955616(86)50031-0

SCHUBERT, G. Stone analysis. Urological Research, v. 34, n. 2, p.146-50, 2006. Disponível em: <https://link.springer.com/article/10.1007\%2Fs00240-005-0028-y>. doi: 10.1007/s00240-005-0028-y

SHAMEMA, A.A.; ARUL, K.T.; KUMAR, R.S.; KALKURA, S.N. Physicochemical analysis of urinary stones from Dharmapuri district. Spectrochimica Acta - Part A Molecular and Biomolecular Spectroscopy, v. 13, n. 1, p. 442-448. Disponível em: <https://doi.org/10.1016/j.saa.2014.05.088>. doi: 10.1016/j.saa.2014.05.088

SIENER, R.; BUCHHOLZ, N.; DAUDON, M.; HESS, B.; KNOLL, T.; OSTHER, P.J.; et al. Quality Assessment of Urinary Stone Analysis: Results of a Multicenter Study of Laboratories in Europe. PLoS One, v. 11, n. 6, p. 1-10, 2016 . Disponível em: < https://journals.plos.org/plosone/article?id=10.1371/journal.pone.0156606>. doi: 10.1371/journal.pone.0156606

TÜRK, C.; PETRIK, A.; SARICA, K.; SEITZ, C.; SKOLARIKOS, A.; et al. EAU Guidelines on diagnosis and conservative management of urolithiasis. European Urology, v. 69, n. 3, p. 468-474, 2016. Disponível em: <https://doi.org/10.1016/j.eururo.2015.07.040>. doi: 10.1016/j.eururo.2015.07.040

ULRICH, L.K.; BIRD, K.A.; KOEHLER, L.A.; SWANSON, L. Urolith analysis. Submission, methods, and interpretation. Veterinary Clinics of North America: Small Animal Practice, v. 26, n. 2, p, 393-400, 1996. Disponível em: <https://doi.org/10.1016/S0195-5616(96)50218-4>. doi: 10.1016/S01955616(96)50218-4

ZHANG, G.M.; SUN, H.; XUE, H.D.; XIAO, H.; ZHANG, X.B.; et al. Prospective prediction of the major component of urinary stone composition with dual-source dual -energy CT in vivo. Clinical Radiology, v.71, n. 11, p. 1178-1183, 2016. Disponível em: <https://doi.org/10.1016/j.crad.2016.07.012>. doi: 10.1016/j.crad.2016.07.012

WEICHSELBAUM, R.C.; FEENEY, D.A.; JESSEN, C.R.; OSBORNE, C.A.; DREYTSER, V.; HOLTE, J. Urocystolith detection: comparison of survey, contrast radiographic and 
ultrasonographic techniques in an in vitro bladder phantom. Veterinary Radiology \& Ultrasound, v. 40, n. 4, p. 386-400, 1999. Disponível em: <https://doi.org/10.1111/j.1740-8261.1999.tb02131.x>. doi: 10.1111/j.17408261.1999.tb02131.x 